\title{
Review Article \\ Current Studies of Acupuncture in Cancer-Induced Bone Pain Animal Models
}

\author{
Hee Kyoung Ryu, Yong-Hyeon Baek, Yeon-Cheol Park, and Byung-Kwan Seo
}

Department of Acupuncture \& Moxibustion, Kyung Hee University Hospital at Gangdong, 149 Sangil-dong, Gangdong-gu, Seoul 134-727, Republic of Korea

Correspondence should be addressed to Byung-Kwan Seo; seohbk@hanmail.net

Received 18 June 2014; Revised 22 August 2014; Accepted 28 August 2014; Published 14 October 2014

Academic Editor: Lixing Lao

Copyright (C) 2014 Hee Kyoung Ryu et al. This is an open access article distributed under the Creative Commons Attribution License, which permits unrestricted use, distribution, and reproduction in any medium, provided the original work is properly cited.

\begin{abstract}
Acupuncture is generally accepted as a safe and harmless treatment option for alleviating pain. To explore the pain mechanism, numerous animal models have been developed to simulate specific human pain conditions, including cancer-induced bone pain (CIBP). In this study, we analyzed the current research methodology of acupuncture for the treatment of CIBP. We electronically searched the PubMed database for animal studies published from 2000 onward using these search terms: (bone cancer OR cancer) AND (pain OR analgesia) AND (acupuncture OR pharmacopuncture OR bee venom). We selected articles that described cancer pain in animal models. We analyzed the methods used to induce cancer pain and the outcome measures used to assess the effects of acupuncture on CIBP in animal models. We reviewed articles that met our inclusion criteria. Injection of mammary cancer cells into the cavity of the tibia was the most frequently used method for inducing CIBP in the animal models. Among the eight selected studies, five studies demonstrated the effects of electroacupuncture on CIBP. The effects of acupuncture were assessed by measuring pain-related behavior. Future researches will be needed to ascertain the effectiveness of acupuncture for treating CIBP and to explore the specific mechanism of CIBP in animal models.
\end{abstract}

\section{Introduction}

Bone pain is a common type of cancer pain [1]. Bone metastasis can occur in advanced cancer, most commonly in breast, prostate, and lung cancer. Bone metastasis can occur in up to 70 percent of patients with advanced breast or prostate cancer and in approximately 40 percent of patients with lung, kidney, or thyroid cancer [2]. Although the exact rate of bone metastasis has not been determined, it was reported that over 400,000 individuals are affected in the United States annually [3].

Tumor growth in bone results in pain, hypercalcemia, anemia, increased risk of infection, pathological fractures, compression of the spinal cord, spinal instability, and decreased mobility. These factors threaten the patient's functional status, quality of life, and survival $[2,4]$. Cancerinduced bone pain (CIBP) is a common symptom in cancer patients, and spontaneous breakthrough pain is severe and difficult to control [1].
The standard management of metastatic CIBP is a combination of radiotherapy and analgesic therapeutics. However, these interventions can fail to provide an adequate analgesic effect [5] and may have side effects. Side effects such as nausea, vomiting, diarrhea, and myelosuppression can occur due to radiotherapy, and constipation, nausea, drowsiness, and cognitive impairment are commonly associated with opioid-based analgesics [6]. Other medications such as calcitonin, bisphosphonate, denosumab, and corticosteroids are also prescribed to alleviate CIBP [7].

Acupuncture may represent a potentially valuable adjunctive strategy for pain alleviation, and it is known to be relatively free of harmful side effects [8]. Guidelines for acupuncture treatment for cancer patients state that acupuncture is effective for postchemotherapy symptoms such as nausea, vomiting, and xerostomia [9]. Additionally, acupuncture has been reported to be helpful for the relief of cancer pain $[10,11]$. The clinical effects of acupuncture on CIBP have not been fully elucidated through rigorous 
randomized controlled trials. Animal models are essential for exploring and understanding the mechanisms of pain and developing effective therapies for its management [12]. Several animal models have been developed and used to investigate CIBP $[13,14]$.

To analyze the current research methodology of acupuncture for the treatment of CIBP, we investigated methods used to induce cancer pain and outcome measures used to assess the effects of acupuncture on cancer pain in various animal models.

\section{Methods}

2.1. Search Strategies. We electronically searched the PubMed database using the terms (bone cancer OR cancer) AND (pain OR analgesia) AND (acupuncture OR pharmacopuncture OR bee venom). Our search was limited to animal studies published from 2000 onward in any language.

We selected articles that described studies of animal cancer pain models. Review articles based on the literature and studies of animal models for other types of disease pain, such as polycystic ovarian syndrome and osteoarthritis, were excluded. We also excluded studies that did not focus on cancer pain and did not use pain-related outcome measures. The full texts of articles meeting the inclusion criteria were obtained and read carefully.

2.2. Data Extraction. The study design data were extracted and classified using a predefined data extraction form that designated the animal used (sex, species, and strain), the CIBP model type (type of cancer cell and site of tumor injection), the intervention type, and the outcome measures (pain-related behavior, macroscopic features, and histological, and biochemical measurements). The data were extracted primarily by one author and were checked by the other authors.

\section{Results}

We retrieved 39 articles and read the titles and abstracts. There was one duplicate record. Of these 38 articles, 10 articles were reviews of the literature and 14 articles were disease studies that were not cancer-related (Figure 1). The remaining 14 articles were reviewed, and six were excluded because they did not focus on cancer pain. Finally, we selected eight animal model studies of CIBP for analysis.

We analyzed the animal models for CIBP in eight studies (Table 1). Five studies used Walker 256 mammary carcinoma cells to induce tumors, and seven studies involved injection of cancer cells into the tibial cavity. Five studies investigated the effects of electroacupuncture on CIBP (Table 2). We also analyzed the outcome measures used in the eight studies [15-22] (Table 3). Five studies assessed pain-related behavior that resulted from CIBP. Among these five studies, three assessed mechanical allodynia or hyperalgesia and four assessed thermal hyperalgesia. Four studies assessed indexes that increased with CIBP or cancer pain and decreased after electroacupuncture treatment $[15,18,20-22]$.

\section{Discussion}

CIBP is a common symptom in cancer patients, and background pain, spontaneous pain, and movement-induced pain are the 3 main characteristics of CIBP. Background pain is a continuous dull ache that increases in intensity as time passes. Severe intermittent pain occurring spontaneously or upon movement or weight bearing is called breakthrough pain [23].

CIBP has a complicated relationship with pathological processes, neuroimmune mediators, tumor growth, and neuropathic conditions [24]. To explore these pain mechanisms, animal models have been developed to simulate specific human pain conditions in order to study the mechanisms and possible therapies [25].

Bone metastases have been classified as osteolytic or osteoblastic. In osteolytic metastases, the destruction of bone is mediated by osteoclasts. Patients with osteolytic metastases have severe pain, pathologic fractures, life-threatening hypercalcemia, spinal cord compression, and other nerve compression syndromes. Osteoblastic metastases can cause bone pain and pathological fractures because of the poor quality of the bone produced by the osteoblasts [26]. Osteolytic metastases occur in approximately 80 percent of patients with stage IV advanced breast cancer, and osteoblastic metastases occur in about 91 percent of patients with advanced prostate cancer [27]. The osteoclastic or osteoblastic pathway is not absolutely dependent on the tumor type because the interaction between osteoclasts and osteoblasts is complex [28]. However, it is clear that the osteoclast is the main factor responsible for bone metastasis pathology [4].

Our results revealed that injection of Walker 256 mammary carcinoma cells into the cavity of the tibia was the most frequently used CIBP animal model [15-19]. Earlier CIBP animal models were based on systemic injection of carcinoma cells. Disseminated malignancy, resulting in more than one randomly sited bone metastasis, occurred with the systemic injection model. In 1999, Schwei et al. [29] described the local injection of osteosarcoma cells into a single bone. At 21 days after the injection of osteolytic sarcoma cells into the cavity of the femur, extensive bone destruction and invasion of the tumor into the periosteum were observed, and these findings were similar to the pathological changes found in patients with osteolytic bone metastases. Thereafter, methods were developed in which breast cancer cells were injected into the tibia of rats, and fibrosarcoma, melanoma $[30,31]$, or adenocarcinoma [31] cells were injected into the humerus or femur of mice. Animals in these models exhibited clinical signs of bone metastasis such as bone destruction, limping, guarding, spontaneous flinching, decreased mobility, secondary hyperalgesia, and allodynia $[30,32]$. These methods produced manifestations similar to human bone cancer symptoms but only partially reflected the pathological process of bone metastasis.

Behavioral tests are used to quantify pain in animals because animals can not directly express their experience of pain. The most commonly used tests are the radiant heat paw-withdrawal test and the von Frey test. The radiant heat paw-withdrawal test is used to assess thermal hyperalgesia. In this test, a noxious stimulus, a high-intensity beam from 
TABLE 1: Models of cancer pain.

\begin{tabular}{lcccccc}
\hline Animal & Cell line & Tumor type & Strain & Sex & Injection site & Reference \\
\hline \multirow{2}{*}{ Rat } & Walker 256 & Mammary gland carcinoma & Wistar & Female & Tibia & {$[15-18]$} \\
& AT-3.1 & Prostate carcinoma & Sprague-Dawley & Female & Tibia & Topenhagen \\
\hline Mouse & S-180 & Sarcoma & Female & Tibia & {$[20,21]$} \\
\hline
\end{tabular}

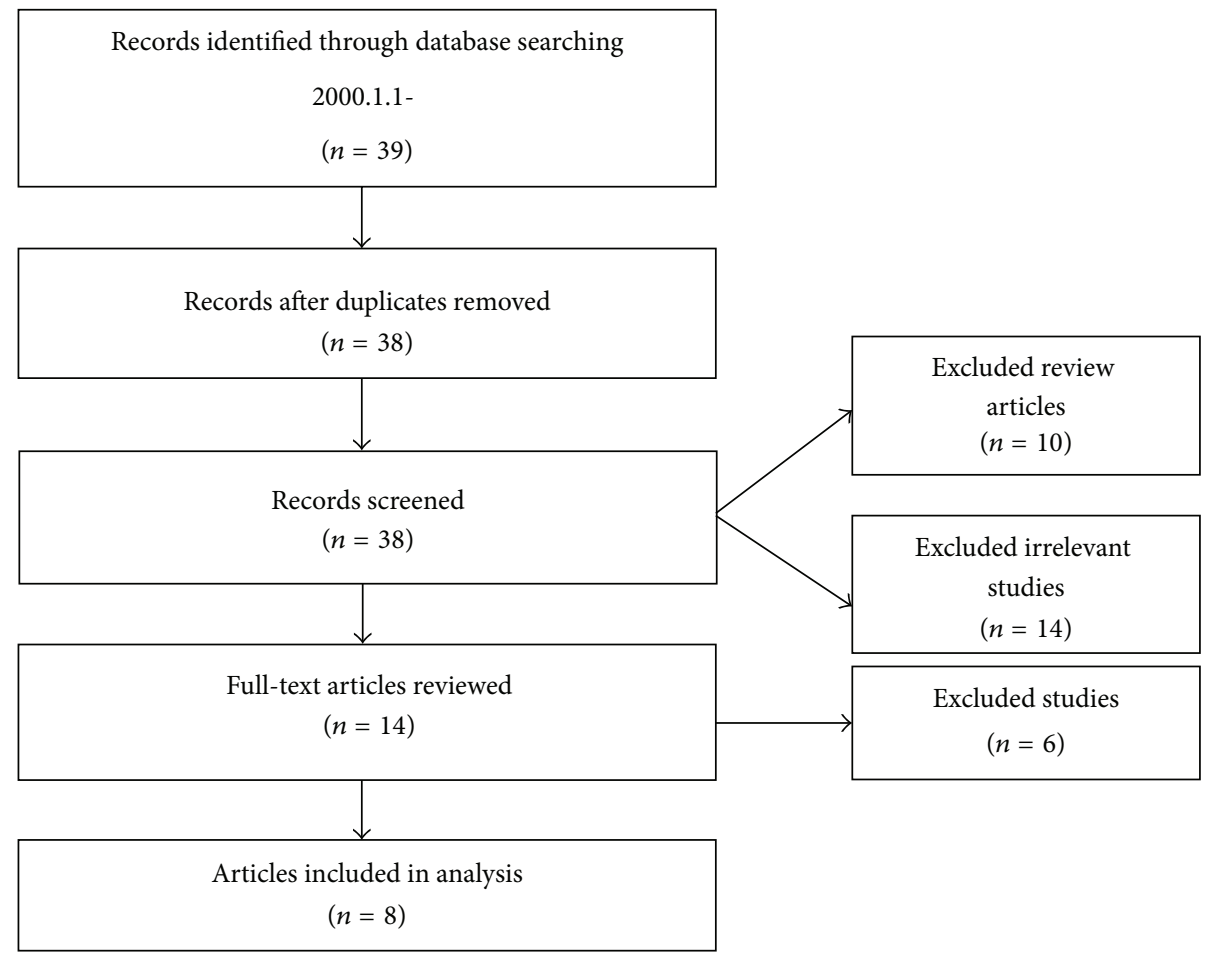

FIGURE 1: Flow diagram of the study selection process.

a projector lamp bulb located below an unheated glass floor, is aimed at the plantar surface of the mid-hind paw. The latency in seconds to withdrawal or pain behavior is measured. Paw withdrawal caused by the stimulation is registered as a response. In the von Frey test, filaments of various thicknesses are applied against the central edge of the hind paw [14]. Increased paw withdrawal latency and filament thickness indirectly signify attenuated hyperalgesia. Furthermore, guarding behaviors such as a particular posture, limping and decreased weight bearing, licking and biting of an affected limb, and behavioral changes are used as indications of spontaneous pain [25]. In spite of the fact that behavioral pain assessments may not reflect the subjective pain experience in humans, these tests have been utilized in various studies. Behavioral assessments have been accepted as easily administrable and highly reproducible maneuvers having detailed and objective parameters that correlate with tumor-induced bone destruction [31].

To determine the mechanism of pain, several studies assessed pain-related factors that were upregulated during cancer pain and were decreased after electroacupuncture.
Zhang et al. [20] used paw withdrawal latency (PWL) and spinal cord interleukin- $\beta$ (IL-1 $\beta$ ) mRNA as outcome measures, thereby identifying the upregulation of spinal IL$1 \beta$ mRNA that induced mechanical and thermal hyperalgesia. Another study [21] evaluated the hind paw withdrawal pressure threshold to assess mechanical hyperalgesia. Furthermore, preprodynorphin (PPD) mRNA and dynorphin were measured in the spinal cord in order to assess the role of dynorphin in cancer pain. Lee et al. [22] detected the upregulation of substance $\mathrm{P}$ during the cancer pain state, as well as increased $\beta$-endorphin production during electroacupuncture-induced analgesia. Kuai et al. [18] evaluated glial fibrillary acidic protein (GFAP). Factors upregulated in the cancer pain state, such as IL- $1 \beta$, PPD mRNA, dynorphin, GFAP, and substance P were decreased after electroacupuncture treatment. This coincided with an improvement in pain-related behavior and suggested that these factors mediate the cancer pain mechanism.

Bisphosphonates are commonly used medications for metastatic bone disease [33]. These agents bind to the surface of the bone and directly cause apoptosis of osteoclasts, inhibit 


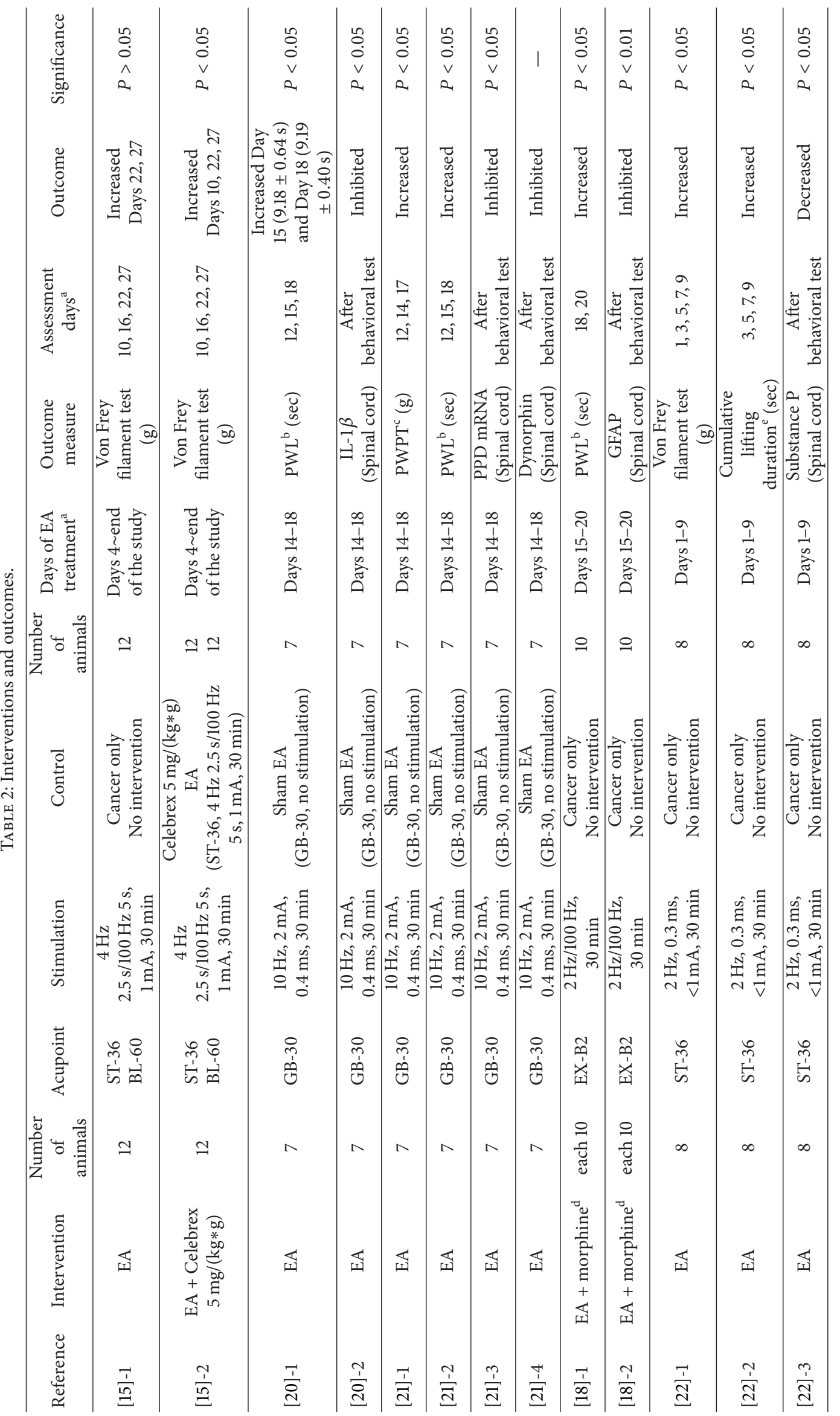




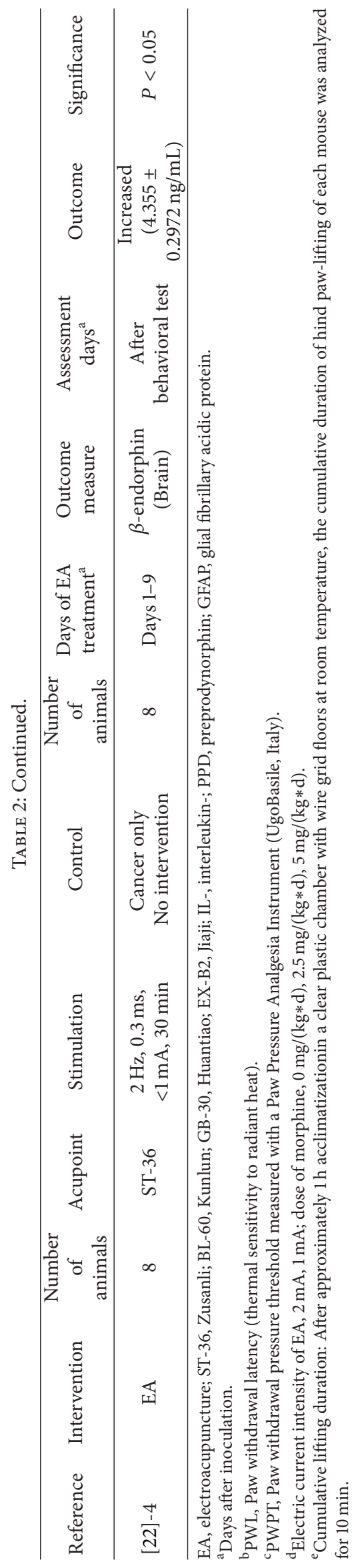


TABLE 3: Outcome measures.

\begin{tabular}{|c|c|c|c|c|c|}
\hline \multirow{2}{*}{ Reference } & \multicolumn{3}{|c|}{ Behavior } & \multirow{2}{*}{ Macroscopicfeatures } & \multirow{2}{*}{$\begin{array}{l}\text { Histological and } \\
\text { biochemical measures (site) }\end{array}$} \\
\hline & $\begin{array}{c}\text { Mechanical stimulus } \\
\text { evoked }\end{array}$ & Heat stimulus evoked & $\begin{array}{l}\text { Movement } \\
\text { related }\end{array}$ & & \\
\hline [15] & $\begin{array}{l}\text { Hind paw withdrawal } \\
\text { (von Frey filaments) }\end{array}$ & & & $\begin{array}{c}\text { Bone surface } \\
\text { Bone destruction } \\
\text { (X-ray) }\end{array}$ & \\
\hline$[20]$ & & $\begin{array}{l}\text { Paw withdrawal latency } \\
\text { (thermal sensitivity to } \\
\text { radiant heat) }\end{array}$ & & & $\begin{array}{c}\text { IL-1 } \beta \\
\text { (spinal cord) }\end{array}$ \\
\hline [21] & $\begin{array}{c}\text { Hind paw withdrawal } \\
\text { pressure threshold } \\
\text { (PWPT) }\end{array}$ & $\begin{array}{l}\text { Paw withdrawal latency } \\
\text { (thermal sensitivity to } \\
\text { radiant heat) }\end{array}$ & & & $\begin{array}{l}\text { PPD mRNA, Dynorphin } \\
\text { (spinal cord) }\end{array}$ \\
\hline$[18]$ & & $\begin{array}{l}\text { Paw withdrawal latency } \\
\text { (thermal sensitivity to } \\
\text { radiant heat) }\end{array}$ & & $\begin{array}{c}\text { Bone surface } \\
\text { Bone destruction } \\
\text { (X-ray) }\end{array}$ & $\begin{array}{c}\text { GFAP } \\
\text { (spinal cord) }\end{array}$ \\
\hline [22] & $\begin{array}{l}\text { Hind paw withdrawal } \\
\text { (von Frey filaments) }\end{array}$ & Cumulative lifting duration & $\begin{array}{l}\text { Clear lifting and } \\
\text { flinching }\end{array}$ & $\begin{array}{l}\text { Tumor size and volume } \\
\text { (MRI scanning) }\end{array}$ & $\begin{array}{c}\text { Substance } \mathrm{P} \\
\text { (spinal cord) } \\
\beta \text {-endorphin } \\
\quad \text { (brain) }\end{array}$ \\
\hline
\end{tabular}

IL-, interleukin-; PPD, preprodynorphin; GFAP, glial fibrillary acidic protein.

osteoclast-mediated bone resorption, and reduce tumorassociated osteolysis [6]. They inhibit tumor cell migration, invasion, adhesion, and cell proliferation, increase tumorassociated macrophages (TAMs), and increase the cytotoxicity of $\gamma \delta$-T cells. Finally, bisphosphonates have shown synergistic antitumor activity with cytotoxic therapies [34, 35]. A recent therapeutic strategy is to target the bone microenvironment, because the complex interplay between cancer cells and the bone microenvironment induces bone metastasis [36].

From our results, outcome measures of pain-related behavior were used to evaluate the cancer pain and its mechanism including allodynia, hyperalgesia, and spontaneous pain. These behavioral responses to stimuli in animals with painful conditions are partially related to the subjective experience of pain in humans. In spite of feasibility and reproducibility of behavioral assessment measure of pain, parameters objectively and directly related to subjective pain experiences in humans describing tumor-induced bone destruction [32]. It is needed to be elucidated the study design to control factors that may exaggerate the effectiveness assessed using pain-related behavioral responses. Blinding maneuver and treatment assignments to prevent assessor bias were elucidated in three studies $[18,20,21]$ while two studies did not described them $[15,22]$. Studies of acupuncture for CIBP using bone microenvironment factors as outcome measures have not been conducted. Bone microenvironment factors such as osteoclasts, osteoblasts, endothelial cells, and hematopoietic progenitor cells may be considered to evaluate the mechanisms of CIBP. It will be required to establish new methods to evaluate pain that are directly related to subjective pain experiences in humans for future research.

This study has limitations stemming from the use of a single database (PubMed) and limited search strategies that returned a small number of studies. Very few studies have been reported on the effect of acupuncture on CIBP. In analyzing the papers in this study, it was difficult to follow a strictly standardized method of analysis because of limited access to complete data from the studies. Future researches are required to fully evaluate the effectiveness of acupuncture and the specific mechanism of CIBP in animal models.

\section{Conflict of Interests}

The authors declare that there is no conflict of interests regarding the publication of this paper.

\section{Authors' Contribution}

BKS is responsible for developing the research strategy and data extraction form, supervising the research fulfilment, and drafting the manuscript. YHB contribute to develop the research strategy and draft the manuscript. HKR is responsible for searching articles, developing data extraction form, extracting data, and drafting the manuscript. YCP contribute to analyze articles and extract data. All authors have read, revised, and approved the final manuscript.

\section{Acknowledgment}

This research was supported by a Grant of the Korea Health Technology R\&D Project through the Korea Health Industry Development Institute (KHIDI), funded by the Ministry of Health \& Welfare, Republic of Korea (Grant no.: HI13C0379).

\section{References}

[1] C. A. Paley, M. I. Bennett, and M. I. Johnson, "Acupuncture for cancer-induced bone pain?" Evidence-Based Complementary 
and Alternative Medicine, vol. 2011, Article ID 671043, 8 pages, 2011.

[2] R. E. Coleman, "Clinical features of metastatic bone disease and risk of skeletal morbidity," Clinical Cancer Research, vol. 12, no. 20, pp. 6243-6249, 2006.

[3] J. M. Jimenez-Andrade, W. G. Mantyh, A. P. Bloom, A. S. Ferng, C. P. Geffre, and P. W. Mantyh, "Bone cancer pain," Annals of the New York Academy of Sciences, vol. 1198, pp. 173-181, 2010.

[4] C. Ripamonti and F. Fulfaro, "Malignant bone pain: pathophysiology and treatments.," Current review of pain, vol. 4, no. 3, pp. 187-196, 2000.

[5] A. Delaney, S. M. Fleetwood-Walker, L. A. Colvin, and M. Fallon, "Translational medicine: cancer pain mechanisms and management," British Journal of Anaesthesia, vol. 101, no. 1, pp. 87-94, 2008.

[6] I. J. Diel, "What do patients with metastatic bone pain need?" European Journal of Cancer, Supplement, vol. 4, no. 8, pp. 1-3, 2006.

[7] S. Buga and J. E. Sarria, "The management of pain in metastatic bone disease," Cancer Control, vol. 19, no. 2, pp. 154-166, 2012.

[8] W. Lu, E. Dean-Clower, A. Doherty-Gilman, and D. S. Rosenthal, "The value of acupuncture in cancer care," Hematology/Oncology Clinics of North America, vol. 22, no. 4, pp. 631$648,2008$.

[9] J. Filshie and J. Hester, "Guidelines for providing acupuncture treatment for cancer patients-a peer-reviewed sample policy document," Acupuncture in Medicine, vol. 24, no. 4, pp. 172-182, 2006.

[10] C. A. Paley, M. I. Johnson, O. A. Tashani, and A.-M. Bagnall, "Acupuncture for cancer pain in adults," Cochrane Database of Systematic Reviews, vol. 19, no. 1, Article ID CD007753, pp. 1-23, 2011.

[11] H. Lee, K. Schmidt, and E. Ernst, "Acupuncture for the relief of cancer-related pain: a systematic review," European Journal of Pain, vol. 9, no. 4, pp. 437-444, 2005.

[12] J. Y. Park, "Animal pain models and behavior tests," Hanyang Medical Reviews, vol. 31, no. 2, pp. 103-106, 2011.

[13] C. A. Paley and M. I. Johnson, "Acupuncture for cancer-induced bone pain: a pilot study," Acupuncture in Medicine, vol. 29, no. 1, pp. 71-73, 2011.

[14] C. Pacharinsak and A. Beitz, "Animal models of cancer pain," Comparative Medicine, vol. 58, no. 3, pp. 220-233, 2008.

[15] Q.-L. Mao-Ying, D.-H. Ren, W.-L. Mi, Q. Liu, and Y.-Q. Wang, "Analgesic effects of electroacupuncture combined with celebrex on rats with tibial cancer pain," Journal of Chinese Integrative Medicine, vol. 6, no. 8, pp. 830-835, 2008.

[16] Q.-L. Mao-Ying, X.-W. Wang, C.-J. Yang et al., "Robust spinal neuroinflammation mediates mechanical allodynia in Walker 256 induced bone cancer rats," Molecular Brain, vol. 5, article $16,2012$.

[17] X. Li, X.-W. Wang, X.-M. Feng, W.-J. Zhou, Y.-Q. Wang, and Q.-L. Mao-Ying, "Stage-dependent anti-allodynic effects of intrathecal Toll-like receptor 4 antagonists in a rat model of cancer induced bone pain," Journal of Physiological Sciences, vol. 63, no. 3, pp. 203-209, 2013.

[18] L. Kuai, H. Chen, T.-T. Zhang, and H.-Y. Yang, "Study on dose-effect relationship of electroacupuncture with different current intensities alleviating tibial cancer pain and inhibition of expression of spinal GFAP in rats," Chinese Acupuncture \& Moxibustion, vol. 32, no. 4, pp. 331-337, 2012.

[19] S. Hu, Q.-L. Mao-Ying, J. Wang et al., "Lipoxins and aspirintriggered lipoxin alleviate bone cancer pain in association with suppressing expression of spinal proinflammatory cytokines," Journal of Neuroinflammation, vol. 9, pp. 278-289, 2012.

[20] R.-X. Zhang, A. Li, B. Liu et al., "Electroacupuncture attenuates bone cancer pain and inhibits spinal interleukin- $1 \beta$ expression in a rat model," Anesthesia and Analgesia, vol. 105, no. 5, pp. 1482-1488, 2007.

[21] R.-X. Zhang, A. Li, B. Liu et al., "Electroacupuncture attenuates bone cancer-induced hyperalgesia and inhibits spinal preprodynorphin expression in a rat model," European Journal of Pain, vol. 12, no. 7, pp. 870-878, 2008.

[22] H.-J. Lee, J.-H. Lee, E.-O. Lee et al., "Substance P and betaendorphin mediate electro-acupuncture induced analgesia in mouse cancer pain model," Journal of Experimental and Clinical Cancer Research, vol. 28, no. 1, pp. 102-110, 2009.

[23] C. Urch, "The pathophysiology of cancer-induced bone pain: current understanding," Palliative Medicine, vol. 18, no. 4, pp. 267-274, 2004.

[24] B. L. Schmidt, D. T. Hamamoto, D. A. Simone, and G. L. Wilcox, "Mechanisms of cancer pain," Molecular Interventions, vol. 10, no. 3, pp. 164-178, 2010.

[25] C. Ma, "Animal models of pain," International Anesthesiology Clinics, vol. 45, no. 2, pp. 121-131, 2007.

[26] G. D. Roodman, "Mechanisms of bone metastasis," The New England Journal of Medicine, vol. 350, no. 16, pp. 1655-1664, 2004.

[27] A. Muralidharan and M. T. Smith, "Pathobiology and management of prostate cancer-induced bone pain: recent insights and future treatments," Inflammopharmacology, vol. 21, no. 5, pp. 339-363, 2013.

[28] M. H. Wong and N. Pavlakis, "Optimal management of bone metastasesin breast cancer patients," Breast Cancer: Targets and Therapy, vol. 3, pp. 35-60, 2011.

[29] M. J. Schwei, P. Honore, S. D. Rogers et al., "Neurochemical and cellular reorganization of the spinal cord in a murine model of bone cancer pain," Journal of Neuroscience, vol. 19, no. 24, pp. 10886-10897, 1999.

[30] P. W. Wacnik, L. J. Kehl, T. M. Trempe, M. L. Ramnaraine, A. J. Beitz, and G. L. Wilcox, "Tumor implantation in mouse humerus evokes movement-related hyperalgesia exceeding that evoked by intramuscular carrageenan," Pain, vol. 101, no. 1-2, pp. 175-186, 2003.

[31] M. A. C. Sabino, N. M. Luger, D. B. Mach, S. D. Rogers, M. J. Schwei, and P. W. Mantyh, "Different tumors in bone each give rise to a distinct pattern of skeletal destruction, bone cancer-related pain behaviors and neurochemical changes in the central nervous system," International Journal of Cancer, vol. 104, no. 5, pp. 550-558, 2003.

[32] S. J. Medhurst, K. Walker, M. Bowes et al., "A rat model of bone cancer pain," Pain, vol. 96, no. 1-2, pp. 129-140, 2002.

[33] G. Hampson and I. Fogelman, "Clinical role of bisphosphonate therapy," International Journal of Women's Health, vol. 4, no. 1, pp. 455-469, 2012.

[34] M. Gnant and P. Clézardin, "Direct and indirect anticancer activity of bisphosphonates: a brief review of published literature," Cancer Treatment Reviews, vol. 38, no. 5, pp. 407-415, 2012. 
[35] P. Clézardin, "Mechanisms of action of bisphosphonates in oncology: a scientific concept evolving from antiresorptive to anticancer activities," BoneKEy Reports, vol. 2, Article ID 267, 2013.

[36] D. F. Camacho and K. J. Pienta, "A multi-targeted approach to treating bone metastases," Cancer and Metastasis Reviews, vol. 33, no. 2-3, pp. 545-553, 2014. 


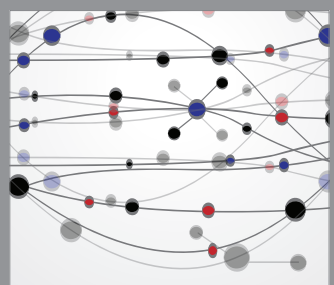

The Scientific World Journal
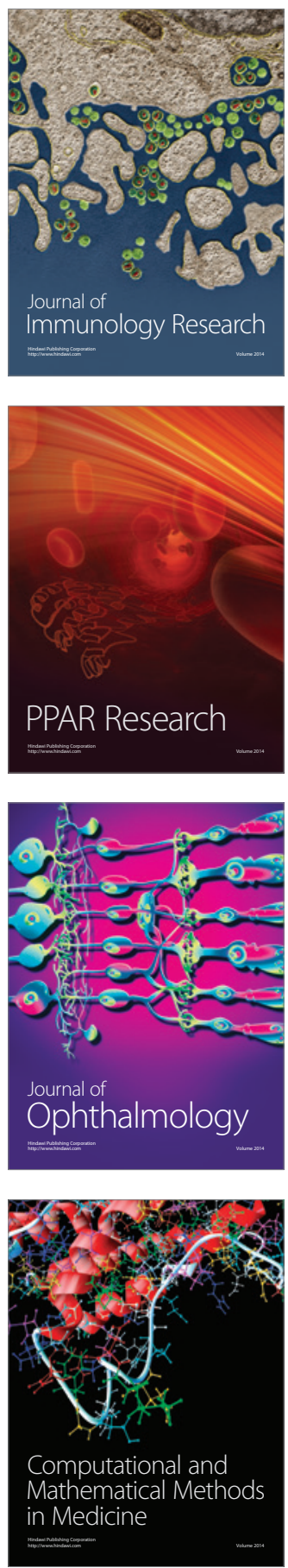

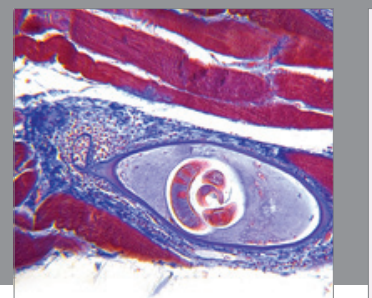

Gastroenterology

Research and Practice
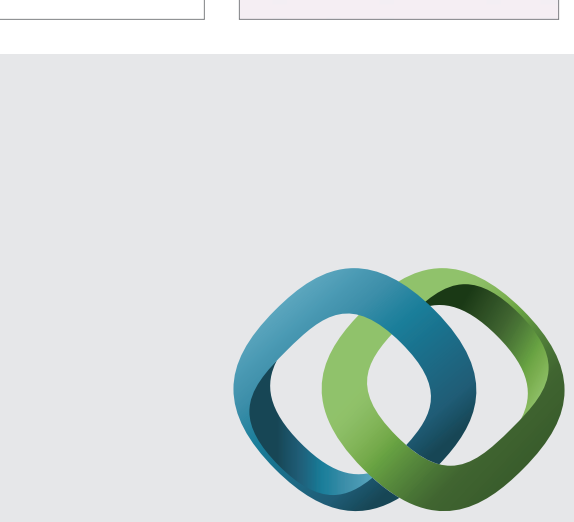

\section{Hindawi}

Submit your manuscripts at

http://www.hindawi.com
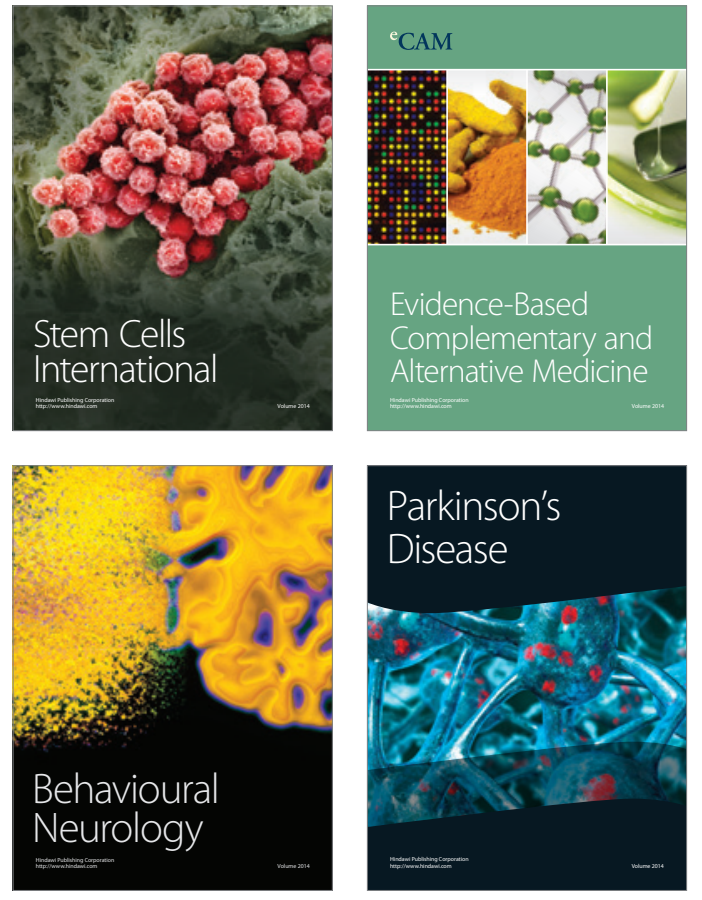
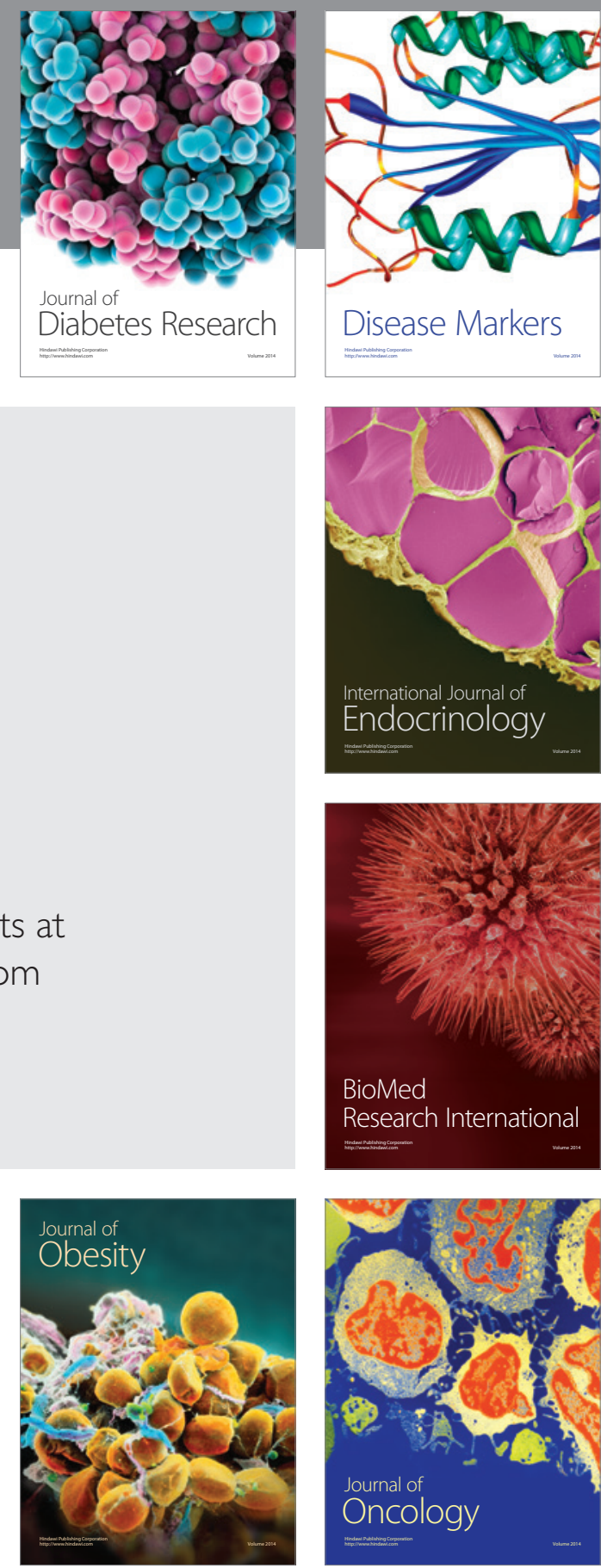

Disease Markers
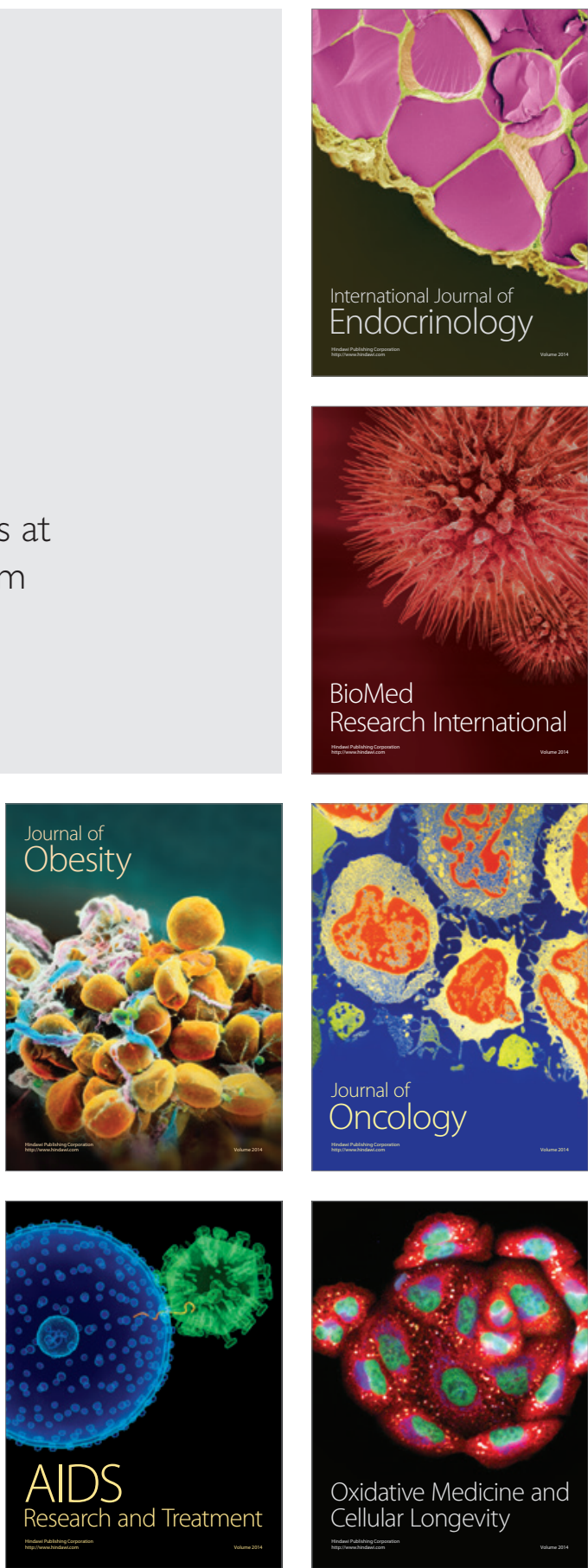